\title{
HAMILTONIAN APPROACHES OF FIELD THEORY
}

\author{
CONSTANTIN UDRIŞTE and ANA-MARIA TELEMAN
}

Received 18 February 2004

\begin{abstract}
We extend some results and concepts of single-time covariant Hamiltonian field theory to the new context of multitime covariant Hamiltonian theory. In this sense, we point out the role of the polysymplectic structure $\delta \otimes J$, we prove that the dual action is indefinite, we find the eigenvalues and the eigenfunctions of the operator $(\delta \otimes J)(\partial / \partial t)$ with periodic boundary conditions, and we obtain interesting inequalities relating functionals created by the new context. As an important example for physics and differential geometry, we study the multitime Yang-Mills-Witten Hamiltonian, extending the Legendre transformation in a suitable way. Our original results are accompanied by well-known relations between Lagrangian and Hamiltonian, and by geometrical explanations regarding the Yang-Mills-Witten Lagrangian.
\end{abstract}

2000 Mathematics Subject Classification: 53C07, 46E20, 55R10.

1. Introduction. As it is well known, the fields theories have a Lagrangian and Hamiltonian structure, since the interactions are mathematically modeled with energetic Lagrangians-Hamiltonians (related by Legendre transformations) and their associated action functionals.

The single-time Hamilton equations appeared for the first time in a paper of Lagrange (1809) on perturbation theory, but it was Cauchy (1831) who first gave the true significance of those equations. In 1835, Hamilton put those equations at the basis of his analytical mechanics and gave the first exact formulation of the least action principle. The theory of single-time Hamilton equations with periodic boundary conditions was initiated by Birkhoff (1917) who proved that the dual action is indefinite. Since then, there were produced a lot of valuable papers that are now summarized in the book of Mawhin and Willem [6].

The multitime Hamilton equations were first written in a book by de Donder [2]. But, to the authors' knowledge, there is no prior work aimed at developing a theory of multitime Hamilton equations with periodic boundary conditions. This is the first subject of the present paper.

Some of the most important Lagrangians are those defined by Yang and Mills (1953) and Witten [18] to produce modern explanations in quantum field theory (Maxwell or Dirac equations). The second subject of the present paper is to introduce and study the (single-time and multitime) Hamiltonian for a Yang-Mills-Witten functional.

Section 1 of this paper contains historical and bibliographical notes. Section 2 (Section 3) recalls the relations between the equations of single-time (multitime) first-order Lagrangian field theory and the covariant Hamilton equations on the finite-dimensional symplectic (polysymplectic) phase space of covariant Hamiltonian field theory. 
Section 4 develops a theory of multitime Hamilton equations with boundary conditions. Section 5 studies the Yang-Mills-Witten Hamiltonian.

2. Single-time Hamilton canonical equations. If $L: \mathbb{R} \times \mathbb{R}^{2 n} \rightarrow \mathbb{R},\left(t, x^{i}, \dot{x}^{i}\right) \rightarrow L\left(t, x^{i}\right.$, $\dot{x}^{i}$ ) is a given Lagrangian, then the associated Euler-Lagrange equations are

$$
\frac{d}{d t} \frac{\partial L}{\partial \dot{x}^{i}}=\frac{\partial L}{\partial x^{i}}, \quad i=1, \ldots, n
$$

Hamilton (1834) simplified the structure of the Euler-Lagrange equations and turned them into a form that has remarkable symmetry by

(1) introducing the conjugate momenta

$$
p_{i}=\frac{\partial L}{\partial \dot{x}^{i}}
$$

(2) considering the Hamiltonian

$$
H=p_{i} \dot{x}^{i}-L
$$

as a function of $t, x, p$.

We suppose that (2.2) defines, for every $x$, a diffeomorphism $\dot{x}^{i} \rightarrow p_{i}$. This map is called the Legendre transform.

THEOREM 2.1. The Euler-Lagrange equations (2.1) are equivalent to the Hamilton equations

$$
\frac{d x^{i}}{d t}=\frac{\partial H}{\partial p_{i}}, \quad \frac{d p_{i}}{d t}=-\frac{\partial H}{\partial x^{i}}, \quad i=1, \ldots, n
$$

Proof. The definitions (2.2) and (2.3) imply

$$
\begin{gathered}
\frac{\partial H}{\partial x^{i}}=p_{j} \frac{\partial \dot{x}^{j}}{\partial x^{i}}-\frac{\partial L}{\partial x^{i}}-\frac{\partial L}{\partial \dot{x}^{j}} \frac{\partial \dot{x}^{j}}{\partial x^{i}}=-\frac{\partial L}{\partial x^{i}}=-\frac{d}{d t} p_{i}, \\
\frac{\partial H}{\partial p_{i}}=\dot{x}^{i}+p_{j} \frac{\partial \dot{x}^{j}}{\partial p_{i}}-\frac{\partial L}{\partial \dot{x}^{j}} \frac{\partial \dot{x}^{j}}{\partial p_{i}}=\dot{x}^{i} .
\end{gathered}
$$

Consequently, the (second-order) ODEs (2.1) are equivalent to (first-order) ODEs (2.4).

2.1. Conservation of the Hamiltonian. Since

$$
\frac{d}{d t} H=\frac{d}{d t}\left(p_{i} \dot{x}^{i}-L\right)=\frac{d p_{i}}{d t} \dot{x}^{i}+p_{i} \frac{d \dot{x}^{i}}{d t}-\frac{\partial L}{\partial t}-\frac{\partial L}{\partial x^{i}} \frac{d x^{i}}{d t}-\frac{\partial L}{\partial \dot{x}^{i}} \frac{d \dot{x}^{i}}{d t}=-\frac{\partial L}{\partial t},
$$

it follows that $H$ is a first integral for the Hamilton equations if and only if the Lagrangian does not depend explicitly on $t$ (autonomous Lagrangian). 
The Hamilton equations can be written in a compact form:

$$
\left(\begin{array}{cc}
0 & \delta_{j}^{i} \\
-\delta_{j}^{i} & 0
\end{array}\right)\left(\begin{array}{c}
\frac{d x^{j}}{d t} \\
\frac{d p_{i}}{d t}
\end{array}\right)+\left(\begin{array}{l}
\frac{\partial H}{\partial x^{i}} \\
\frac{\partial H}{\partial p_{i}}
\end{array}\right)=\left(\begin{array}{l}
0 \\
0
\end{array}\right)
$$

where $J=\left(\begin{array}{cc}0 & \delta_{j}^{i} \\ -\delta_{j}^{i} & 0\end{array}\right)$ is the symplectic (complex) structure on $\mathbb{R}^{2 n}$.

The minimization of the dual single-time action, the eigenvalues and the eigenfunctions of $J(d / d t)$ with periodic boundary conditions, and existence of periodic solutions of single-time Hamilton equations were discussed in [6].

3. Multitime Hamilton canonical equations. Let $L: \mathbb{R}^{p} \times \mathbb{R}^{n} \times \mathbb{R}^{n p} \rightarrow \mathbb{R},\left(t^{\alpha}, x^{i}, x_{\alpha}^{i}\right) \rightarrow$ $L\left(t^{\alpha}, x^{i}, x_{\alpha}^{i}\right), x_{\alpha}^{i}=\partial x^{i} / \partial t^{\alpha}$, be a given Lagrangian. The associated Euler-Lagrange equations are

$$
\frac{\partial}{\partial t^{\alpha}} \frac{\partial L}{\partial x_{\alpha}^{i}}=\frac{\partial L}{\partial x^{i}}, \quad i=1, \ldots, n, \alpha=1, \ldots, p
$$

In [2], de Donder turned these equations into a form that has remarkable symmetry. He used

(1) the conjugate momenta

$$
p_{k}^{\alpha}=\frac{\partial L}{\partial x_{\alpha}^{k}}
$$

(2) the Hamiltonian

$$
H=p_{k}^{\alpha} x_{\alpha}^{k}-L
$$

as a function of $t^{\alpha}, x^{i}, p_{i}^{\alpha}$.

Here it is, of course, required that (3.2) defines, for every $x=\left(x^{i}\right)$, a continuously differentiable bijection $x_{\alpha}^{i} \rightarrow p_{i}^{\alpha}$. This map is called the Legendre transform.

THEOREM 3.1. The Euler-Lagrange equations (3.1) are equivalent to the Hamilton equations

$$
\frac{\partial x^{i}}{\partial t^{\alpha}}=\frac{\partial H}{\partial p_{i}^{\alpha}}, \quad \frac{\partial p_{i}^{\alpha}}{\partial t^{\alpha}}=-\frac{\partial H}{\partial x^{i}}, \quad i=1, \ldots, n, \alpha=1, \ldots, p
$$

(summation over $\alpha$ ).

Proof. The definitions (3.2) and (3.3) imply

$$
\begin{gathered}
\frac{\partial H}{\partial x^{i}}=p_{k}^{\alpha} \frac{\partial x_{\alpha}^{k}}{\partial x^{i}}-\frac{\partial L}{\partial x^{i}}-\frac{\partial L}{\partial x_{\alpha}^{k}} \frac{\partial x_{\alpha}^{k}}{\partial x^{i}}=-\frac{\partial L}{\partial x^{i}}=-\frac{\partial p_{i}^{\alpha}}{\partial t^{\alpha}}, \\
\frac{\partial H}{\partial p_{i}^{\alpha}}=x_{\alpha}^{i}+p_{k}^{\beta} \frac{\partial x_{\beta}^{k}}{\partial p_{i}^{\alpha}}-\frac{\partial L}{\partial x_{\beta}^{k}} \frac{\partial x_{\beta}^{k}}{\partial p_{i}^{\alpha}}=x_{\alpha}^{i} .
\end{gathered}
$$

The Euler-Lagrange (second-order) PDEs (3.1) are equivalent to Hamilton (first-order) PDEs (3.4). 
3.1. Conservation of the energy-momentum tensor field. The energy-momentum tensor field is defined by

$$
T_{\beta}^{\alpha}=x_{\beta}^{i} \frac{\partial L}{\partial x_{\alpha}^{i}}-\delta_{\beta}^{\alpha} L
$$

We compute the divergence of this tensor field. We find

$$
\begin{aligned}
\frac{\partial}{\partial t^{\alpha}} T_{\beta}^{\alpha} & =\frac{\partial}{\partial t^{\alpha}}\left(x_{\beta}^{i} p_{i}^{\alpha}\right)-\delta_{\beta}^{\alpha}\left(\frac{\partial L}{\partial t^{\alpha}}+\frac{\partial L}{\partial x^{i}} x_{\alpha}^{i}+\frac{\partial L}{\partial x_{\gamma}^{i}} \frac{\partial x_{\gamma}^{i}}{\partial t^{\alpha}}\right) \\
& =\frac{\partial x_{\beta}^{i}}{\partial t^{\alpha}} p_{i}^{\alpha}+x_{\beta}^{i} \frac{\partial p_{i}^{\alpha}}{\partial t^{\alpha}}-\frac{\partial L}{\partial t^{\beta}}-\frac{\partial L}{\partial x^{i}} x_{\beta}^{i}-\frac{\partial L}{\partial x_{\gamma}^{i}} \frac{\partial x_{\gamma}^{i}}{\partial t^{\beta}} \\
& =x_{\beta}^{i}\left(\frac{\partial p_{i}^{\alpha}}{\partial t^{\alpha}}-\frac{\partial L}{\partial x^{i}}\right)-\frac{\partial L}{\partial t^{\beta}}=-\frac{\partial L}{\partial t^{\beta}} .
\end{aligned}
$$

Consequently, the energy-momentum tensor field is conserved if and only if the Lagrangian $L$ does not depend explicitly on $t^{\alpha}$ (autonomous Lagrangian).

4. Minimization of dual multitime action. The Hamilton equations can be written in the form

$$
\delta_{\beta}^{\alpha} \delta_{j}^{i} \frac{\partial p_{i}^{\beta}}{\partial t^{\alpha}}+\frac{\partial H}{\partial x^{j}}=0, \quad-\delta_{\beta}^{\alpha} \delta_{j}^{i} \frac{\partial x^{j}}{\partial t^{\alpha}}+\frac{\partial H}{\partial p_{i}^{\beta}}=0 .
$$

We will use also the polysymplectic structure

$$
\delta \otimes J=\left(\begin{array}{cc}
0 & \delta_{\beta}^{\alpha} \delta_{j}^{i} \\
-\delta_{\beta}^{\alpha} \delta_{j}^{i} & 0
\end{array}\right), \quad \delta=\left(\delta_{\beta}^{\alpha}\right), \quad J=\left(\begin{array}{cc}
0 & \delta_{j}^{i} \\
-\delta_{j}^{i} & 0
\end{array}\right)
$$

discovered by the first author $[13,16,17]$. In this sense, we introduce the notations

$$
\begin{gathered}
X=\mathbb{R}^{n}, \quad X^{*}=\text { dual of } X, \quad Y=\mathbb{R}^{p}, \quad Y^{*}=\text { dual of } Y, \\
\delta=i d_{X \otimes Y}, \quad \delta \otimes J=\left(\begin{array}{cc}
0 & \varphi(\delta) \\
-\vartheta(\delta) & 0
\end{array}\right),
\end{gathered}
$$

where $\varphi, \vartheta$ are the canonical isomorphisms:

$$
\begin{gathered}
\varphi: \operatorname{End}(X \otimes Y) \longrightarrow \operatorname{Hom}\left(X^{*} \otimes \operatorname{End} Y, X^{*}\right) \\
\vartheta: \operatorname{End}(X \otimes Y) \longrightarrow \operatorname{Hom}\left(X \otimes Y^{*}, X \otimes Y^{*}\right) .
\end{gathered}
$$

Then

$$
\delta \otimes J \in \operatorname{Hom}\left(\left(X \otimes Y^{*}\right) \oplus\left(X^{*} \otimes \operatorname{End} Y\right), X^{*} \oplus\left(X \otimes Y^{*}\right)\right)
$$

and we can give the Hamilton equations the following more compact form:

$$
(\delta \otimes J)\left(\begin{array}{c}
\frac{\partial x}{\partial t} \\
\frac{\partial p}{\partial t}
\end{array}\right)=-\left(\begin{array}{c}
\frac{\partial H}{\partial x} \\
\frac{\partial H}{\partial p}
\end{array}\right) .
$$


According to the standard Riemannian metric on $\mathbb{R}^{n+n p}$, which is given by the matrix $G=\left(\begin{array}{cc}\delta_{i j} & 0 \\ 0 & \delta^{\alpha \beta} \delta_{i j}\end{array}\right)$, the associated Hamilton multitime action $\psi$ is given by

$$
\psi(u)=\int_{\Omega} \mathscr{L}\left(t, u, u^{\prime}\right) d v, \quad \Omega=[0, T]^{p} \subset \mathbb{R}^{p}, \quad T>0
$$

where $u=(x, p), u^{\prime}=\partial u / \partial t, d v=d t^{1} \wedge \cdots \wedge d t^{p}$, and

$$
\begin{aligned}
\mathscr{L}\left(t, u, u^{\prime}\right) & =\frac{1}{2}\left(\frac{\partial p_{i}^{\alpha}}{\partial t^{\alpha}} x^{i}-\frac{\partial x^{i}}{\partial t^{\alpha}} p_{i}^{\alpha}\right)+H(t, x, p) \\
& =\frac{1}{2}\left(\frac{\partial p_{j}^{\alpha}}{\partial t^{\alpha}},-\frac{\partial x^{j}}{\partial t^{\beta}}\right)\left(\begin{array}{cc}
\delta_{i j} & 0 \\
0 & \delta^{\beta \alpha} \delta_{i j}
\end{array}\right)\left(\begin{array}{c}
x^{i} \\
p_{i}^{\alpha}
\end{array}\right)+H(t, x(t), p(t)) .
\end{aligned}
$$

As it is well known, the Euler-Lagrange equations associated with the action $\psi$ are equivalent to (3.4). The action $\psi$ is indefinite. This is easily shown by substituting the function $u$ with the sequence of functions

$$
\begin{gathered}
u_{k}=\left(x_{k}, p_{k}\right), \quad x_{k}^{i}(t)=c^{i} \cos \left(\lambda_{k} t^{\beta}\right), \quad p_{k, i}^{\alpha}(t)=c_{i}^{\alpha} \sin \left(\lambda_{k} t^{\beta}\right), \\
u_{k}(t)=\cos \left(\lambda_{k} t^{\beta}\right)(\delta \otimes I) c-\sin \left(\lambda_{k} t^{\beta}\right)(\delta \otimes J) c, \quad \beta=\text { fixed }
\end{gathered}
$$

into the previous integral; we choose $\lambda_{k}=2 k \pi / T, k \in \mathbb{Z}, c=\left(c^{i}, c_{i}^{\alpha}\right) \in \mathbb{R}^{n+n p},\|c\|=1$. It follows that

$$
\begin{gathered}
\left\|u_{k}(t)\right\|=1 \\
\left(\frac{\partial p_{i}^{\alpha}}{\partial t^{\alpha}},-\frac{\partial x^{i}}{\partial t^{\mu}}\right)\left(\begin{array}{cc}
\delta_{i j} & 0 \\
0 & \delta^{\mu \gamma} \delta_{i j}
\end{array}\right)\left(\begin{array}{l}
x^{j} \\
p_{j}^{\gamma}
\end{array}\right)=\lambda_{k} c_{j}^{\beta} c^{j} \quad \forall k \in \mathbb{Z} .
\end{gathered}
$$

Consequently,

$$
\psi\left(u_{k}\right)=\frac{k \pi}{T} T^{p} c_{i}^{\beta} c^{i}+\int_{\Omega}\left(t, u_{k}(t)\right) d v \longrightarrow-\infty \text { or }+\infty
$$

according to $k \rightarrow-\infty$ or $k \rightarrow+\infty$. That is why the direct method of the calculus of variations cannot be applied directly and more sophisticated approaches like minimax methods, isoperimetric natural constraints, or dual least action principles have to be used. In a forthcoming paper, we will concentrate on the case where the Hamiltonian $H(t, u)$ is convex in $u$, in which case the dual least action principle seems to provide the best results in the simplest way. Here we discuss the eigenvalues and the eigenfunctions of the operator $(\delta \otimes J)(\partial / \partial t)$ with periodic boundary conditions:

$$
(\delta \otimes J)\left(\frac{\partial u}{\partial t}\right)=\lambda u(t), \quad u(0, \ldots, 0)=u\left(k_{1} T, \ldots, k_{p} T\right),
$$

where $\lambda \in \mathbb{R}, \partial u / \partial t$ is the matrix of elements $\partial u / \partial t^{\alpha}$, and $k_{\alpha} \in \mathbb{Z}$. The PDEs system (4.12) is equivalent to

$$
\frac{\partial x^{i}}{\partial t^{\alpha}}=-\lambda p_{i}^{\alpha}, \quad \frac{\partial p_{i}^{\alpha}}{\partial t^{\alpha}}=\lambda x^{i}
$$


We get particular solutions by taking

$$
x^{i}(t)=c^{i} \cos \left(\lambda_{\alpha}^{i} t^{\alpha}\right), \quad p_{i}^{\beta}=c_{i}^{\beta} \sin \left(\mu_{i \alpha} t^{\alpha}\right),
$$

where $\lambda_{\alpha}^{i}, \mu_{i \alpha}, c^{i}, c_{i}^{\beta}$ are real numbers such that

$$
\frac{T \lambda_{\alpha}^{i}}{2 \pi} \in \mathbb{Z}, \quad \lambda_{\alpha}^{i}=\mu_{i \alpha}, \quad c^{i} \lambda_{\alpha}^{i}=\lambda c_{i}^{\alpha}, \quad c_{i}^{\alpha} \mu_{i \alpha}=\lambda c^{i} .
$$

Supposing that $c^{i} \neq 0$, we get

$$
\lambda^{2}=\delta^{\alpha \beta} \lambda_{\alpha}^{i} \lambda_{\beta}^{i}, \quad i=1, \ldots, n .
$$

We have the following theorem.

THEOREM 4.1. The system (4.12) has a nontrivial solution of the form (4.14) if and only if the equation $T^{2} \lambda^{2}=4 \pi^{2} \delta^{\alpha \beta} k_{\alpha} k_{\beta}$ has solutions $\left(k_{1}, \ldots, k_{p}\right) \in \mathbb{Z}^{p}$.

NoTE 4.2. The PDEs system (4.12) is associated with the Lagrangian

$$
L_{1}\left(x, \frac{\partial x}{\partial t}\right)=\frac{\lambda}{2}\left(\delta_{i j} x^{i} x^{j}+\delta^{\alpha \beta} \delta_{i j} x_{\alpha}^{i} x_{\beta}^{j}\right)
$$

We could consider the Hamiltonian

$$
H_{2}(x, p)=\frac{1}{2}\left[g_{i j} x^{i} x^{j}+G_{\alpha \beta}^{i j} p_{i}^{\alpha} p_{j}^{\beta}\right],
$$

with real and constant coefficients $g_{i j}, G_{\alpha \beta}^{i j}$. This generalizes the Hamiltonian associated with the linear oscillator. The associated PDEs system will be linear and with constant coefficients:

$$
\frac{\partial x^{i}}{\partial t^{\alpha}}=G_{\alpha \beta}^{i j} p_{j}^{\beta}, \quad \frac{\partial p_{i}^{\alpha}}{\partial t^{\alpha}}=-g_{i j} x^{j}
$$

When we look for solutions of the form

$$
x^{i}(t)=c^{i} \exp \left(\lambda_{\alpha} t^{\alpha}\right), \quad p_{i}^{\beta}(t)=c_{i}^{\beta} \exp \left(\lambda_{\alpha} t^{\alpha}\right)
$$

with constants $c^{i}, c_{i}^{\beta}$, we obtain the algebraic equations

$$
\lambda_{\alpha} c^{i}=G_{\alpha \beta}^{i j} c_{j}^{\beta}, \quad \lambda_{\alpha} c_{i}^{\alpha}=-g_{i j} c^{j} .
$$

Supposing that $\operatorname{det}\left(g_{i j}\right) \neq 0$, we get further

$$
c^{j}=-g^{i j} c_{i}^{\alpha} \lambda_{\alpha}, \quad\left(G_{\alpha \beta}^{i j}+g^{i j} \lambda_{\alpha} \lambda_{\beta}\right) c_{i}^{\alpha}=0 .
$$

REMARK 4.3. Since the set of eigenvalues $\lambda$ of the differential operator $\delta \otimes J$ is unbounded (from below and from above), the quadratic form

$$
u \rightarrow \frac{1}{2} \int_{\Omega}\left\langle(\delta \otimes J) \frac{\partial u}{\partial t}(t), u(t)\right\rangle d v
$$


is indefinite on the space

$$
\begin{aligned}
H_{\Omega}^{1}=\{ & u: \mathbb{R}^{p} \longrightarrow \mathbb{R}^{n+n p} \mid u=\text { absolutely continuous, } \\
& \left.k_{\alpha} \in \mathbb{Z} \Longrightarrow u\left(t^{1}, \ldots, t^{p}\right)=u\left(t^{1}+k_{1} T, \ldots, t^{p}+k_{p} T\right), \frac{\partial u}{\partial t} \in L^{2}\left(\mathbb{R}^{p}\right)\right\} .
\end{aligned}
$$

THEOREM 4.4. If $u \in H_{\Omega}^{1}$, then

$$
\int_{\Omega}\|u(t)\|^{2} d v \leq \frac{T^{2}}{4 \pi^{2}} \int_{\Omega}\left\|\frac{\partial u}{\partial t}(t)\right\|^{2} d v .
$$

Proof. By assumption, the function $u$ has a Fourier expansion

$$
u(t)=\sum_{\left(k_{1}, \ldots, k_{p}\right) \in \mathbb{Z}^{p}} c\left(k_{1}, \ldots, k_{p}\right) \exp \left(2 \pi i \frac{k_{1} t_{1}+\cdots+k_{p} t_{p}}{T}\right) .
$$

If we denote $k=\left(k_{1}, \ldots, k_{p}\right)$, then $\|k\|^{2}=k_{1}^{2}+\cdots+k_{p}^{2}$. The Perseval equality implies

$$
\begin{aligned}
\int_{\Omega}\left\|\frac{\partial u}{\partial t}\right\|^{2} d v & =\sum_{k_{1}, \ldots, k_{p}} T^{p} \frac{4 \pi^{2}\|k\|^{2}}{T^{2}}\left\|c\left(k_{1}, \ldots, k_{p}\right)\right\|^{2} \\
& \geq \frac{4 \pi^{2}}{T^{2}} \sum_{k_{1}, \ldots, k_{p}} T^{p}\left\|\left(k_{1}, \ldots, k_{p}\right)\right\|^{2}=\frac{4 \pi^{2}}{T^{2}} \int_{\Omega}\|u(t)\|^{2} d v .
\end{aligned}
$$

THEOREM 4.5. Every $u \in H_{\Omega}^{1}$ satisfies the inequality

$$
\int_{\Omega}\left\langle(\delta \otimes J) \frac{\partial u}{\partial t}, u(t)\right\rangle d v \geq-\frac{\sqrt{p} T}{2 \pi} \int_{\Omega}\left\|\frac{\partial u}{\partial t}(t)\right\| d v .
$$

Proof. We use the deviation

$$
\tilde{u}(t)=u(t)-\frac{1}{T^{p}} \int_{\Omega} u(t) d v .
$$

The Cauchy-Schwarz inequality and inequality (4.25) imply

$$
\begin{aligned}
\int_{\Omega}\langle & \left.(\delta \otimes J) \frac{\partial u}{\partial t}, u(t)\right\rangle d v=\int_{\Omega}\left\langle(\delta \otimes J) \frac{\partial u}{\partial t}, \tilde{u}(t)\right\rangle d v \\
& \geq-\left(\int_{\Omega}\left\|(\delta \otimes J) \frac{\partial u}{\partial t}(t)\right\|^{2} d v\right)^{1 / 2}\left(\int_{\Omega}\|\tilde{u}(t)\|^{2} d v\right)^{1 / 2} \\
& \geq-\frac{T}{2 \pi}\left(\int_{\Omega}\left\|(\delta \otimes J) \frac{\partial u}{\partial t}(t)\right\|^{2} d v\right)^{1 / 2}\left(\int_{\Omega}\left\|\frac{\partial \tilde{u}}{\partial t}(t)\right\|^{2} d v\right)^{1 / 2} \\
& \geq-\frac{\sqrt{p} T}{2 \pi} \int_{\Omega}\left\|\frac{\partial u}{\partial t}(t)\right\|^{2} d v .
\end{aligned}
$$

5. Witten-type Hamiltonians. The study of the Yang-Mills-Witten-type functionals $[1,3,4,5,7,8,9,10,11,12,14,15]$, which was initially related to a modern approach of the Maxwell or Dirac equations, gave extremely important results for the development of the differential geometry. This theory which is both experimentally and theoretically grounded, became very interesting and with many possible applications and open 
problems for research. In this section, we will apply the Hamiltonian formalism to a functional of this type.

Let $\operatorname{Spin}^{c}(4)=\operatorname{Spin}(4) \times_{\mathbb{Z}_{2}} S^{1}=[\operatorname{SU}(2) \times \operatorname{SU}(2)] \times_{\mathbb{Z}_{2}} S^{1}$. There is a natural identification

$$
\operatorname{Spin}^{c}(4) \equiv\left\{\left(a_{+}, a_{-}\right) \in U(2) \times U(2) ; \operatorname{det}\left(a_{+}\right)=\operatorname{det}\left(a_{-}\right)\right\},
$$

which induces the projections

$$
\varrho_{ \pm}: \operatorname{Spin}^{c}(4) \longrightarrow U(2)
$$

and also the morphism

$$
\operatorname{det}: \operatorname{Spin}^{c}(4) \rightarrow S^{1}
$$

defined by $\operatorname{det}\left(a_{+}, a_{-}\right)=\operatorname{det}\left(a_{+}\right)=\operatorname{det}\left(a_{-}\right)$for any $\left(a_{+}, a_{-}\right) \in \operatorname{Spin}^{c}(4)$. Let $(X, g)$ be a Riemannian, compact, and oriented 4-manifold. Let $\left(P^{c}, \gamma\right)$ be a $\operatorname{Spin}^{c}(4)$-structure in $(X, g)$ and $\Sigma^{ \pm}=P^{c} \times \varrho_{ \pm} C^{2}$, its associated spinor-vector bundles.

We will consider as well the complex line bundle $\operatorname{det} \Sigma^{+} \equiv \operatorname{det} \Sigma^{-} \equiv P^{c} \times \operatorname{det} C$ and also the space of the unitary connections in $\operatorname{det} \Sigma^{+}$, denoted by $\mathscr{A}\left(\operatorname{det} \Sigma^{+}\right)$. The space $\mathscr{A}\left(\operatorname{det} \Sigma^{+}\right)$has a natural structure of affine space modeled over the vector space $i A^{1}(X)$, where $A^{1}(X)$ is the space of the $C^{\infty}-1$-forms defined on the manifold $X$. Let

$$
\begin{gathered}
W: \mathscr{A}\left(\operatorname{det} \Sigma^{+}\right) \times A^{0}\left(\Sigma^{+}\right) \longrightarrow \mathbb{R} \\
W(A, \psi)=\left\|\nabla_{\hat{A}} \psi\right\|^{2}+\frac{1}{16}\left\|F_{A}\right\|^{2}+\frac{1}{4}\|\psi\|_{L^{4}}^{4}+\frac{1}{4} \int_{X} s|\psi|^{2}
\end{gathered}
$$

be the Witten-type functional, where $(A, \psi) \in \mathscr{C}=\mathscr{A}\left(\operatorname{det} \Sigma^{+}\right) \times A^{0}\left(\Sigma^{+}\right),|\psi|^{2}=\psi \bar{\psi}$, the norms $\|\cdot\|$ are $L^{2}$-norms, and $\hat{A}$ is the connection naturally induced in $\Sigma^{+}$by $A$ and by the Levi-Civita connection of $(X, g)$.

We assume that the restriction of the Riemannian structure $g$ to a coordinate neighborhood is Euclidean and we make our computations only over this neighborhood. It follows that the Levi-Civita connection associated to $g$ has its coefficients, the curvature, and its scalar curvature all equal to 0 . Under such conditions, we apply the single-time or multitime Hamiltonian formalism, and we will use the symbol sigma with indices for summation.

If the connection $A$ has its local associated form $i a$, then $\nabla_{\hat{A}} \psi=d \psi+(1 / 2) i a \psi$ for any $\psi \in A^{0}\left(\Sigma^{+}\right)$. Of course, $\psi=\left(\psi^{1}, \psi^{2}\right)=\left(\varphi^{1}+i \eta^{1}, \varphi^{2}+i \eta^{2}\right)$ and $a=a_{\alpha} d t^{\alpha}$.

LEMMA 5.1. The Lagrangian associated to $W$ is given by the formula

$$
\begin{aligned}
L(A, \psi)= & \sum_{\alpha=0}^{3} \sum_{j=1}^{2}\left[\frac{1}{4}\left(a_{\alpha}\right)^{2}\left(\left(\varphi^{j}\right)^{2}+\left(\eta^{j}\right)^{2}\right)+\left(\varphi_{\alpha}^{j}\right)^{2}+\left(\eta_{\alpha}^{j}\right)^{2}+\left(\varphi^{j} \eta_{\alpha}^{j}-\eta^{j} \varphi_{\alpha}^{j}\right) a_{\alpha}\right] \\
& +\frac{1}{32} \sum_{\alpha, \beta=0}^{3}\left(a_{\alpha, \beta}-a_{\beta, \alpha}\right)^{2}+\frac{1}{4}\left(\sum_{j=1}^{2}\left[\left(\varphi^{j}\right)^{2}+\left(\eta^{j}\right)^{2}\right]\right)^{2}
\end{aligned}
$$

for any $\psi=\left(\varphi^{1}+i \eta^{1}, \varphi^{2}+i \eta^{2}\right) \in A^{0}\left(\Sigma^{+}\right), a=a_{\alpha} d t^{\alpha}$. 
5.1. Single-time Hamilton equations. We can assume, under a suitable gauge transformation, that $a_{0}=0$.

LEMMA 5.2. The momenta associated to L are

$$
p^{\alpha}=\frac{\partial L}{\partial a_{\alpha, 0}}=\frac{1}{8} a_{\alpha, 0}, \quad q_{j}=\frac{\partial L}{\partial \varphi_{0}^{j}}=2 \varphi_{0}^{j}, \quad r_{j}=\frac{\partial L}{\partial \eta_{0}^{j}}=2 \eta_{0}^{j},
$$

with $\alpha=1,2,3$ and $j=1,2$.

LEMMA 5.3. The Hamilton density associated to $W$ is

$$
\begin{aligned}
H= & 4 \sum_{s=1}^{3}\left(p^{s}\right)^{2}+\frac{1}{4} \sum_{j=1}^{2}\left[q_{j}^{2}+r_{j}^{2}\right] \\
& -\sum_{s=1}^{3} \sum_{j=1}^{2}\left[\frac{1}{4}\left(a_{s}\right)^{2}\left(\left(\varphi^{j}\right)^{2}+\left(\eta^{j}\right)^{2}\right)+\left(\varphi_{s}^{j}\right)^{2}+\left(\eta_{s}^{j}\right)^{2}+\left(\varphi^{j} \eta_{s}^{j}-\eta^{j} \varphi_{s}^{j}\right) a_{s}\right] \\
& -\frac{1}{16} \sum_{s, t=1}^{3}\left[\left(a_{s, t}\right)^{2}-a_{s, t} a_{t, s}\right]-\frac{1}{4}\left(\sum_{j=1}^{2}\left[\left(\varphi^{j}\right)^{2}+\left(\eta^{j}\right)^{2}\right]\right)^{2} .
\end{aligned}
$$

THEOREM 5.4. The Hamilton generalized equations, associated to $W$, are

$$
\begin{gathered}
\dot{a}_{s}=8 p^{s}, \quad \dot{\varphi}^{j}=\frac{1}{2} q_{j}, \quad \dot{\eta}^{j}=\frac{1}{2} r_{j}, \\
\dot{p}^{s}=\frac{1}{2} a_{s}|\psi|^{2}+\sum_{j=1}^{2}\left(\varphi^{j} \eta_{s}^{j}-\eta^{j} \varphi_{s}^{j}\right)+\frac{1}{8} \sum_{t=1}^{3}\left(a_{s, t t}-a_{t, s t}\right), \\
\dot{q}_{j}=|\psi|^{2} \varphi^{j}+\sum_{t=1}^{3}\left(\frac{1}{2}\left(a_{t}\right)^{2} \varphi^{j}+2 \varphi_{t t}^{j}-a_{t, t} \eta^{j}\right), \\
\dot{r}_{j}=|\psi|^{2} \eta^{j}+\sum_{t=1}^{3}\left(\frac{1}{2}\left(a_{t}\right)^{2} \eta^{j}+2 \eta_{t t}^{j}+a_{t, t} \varphi^{j}\right), \quad j=1,2 ; s=1,2,3 .
\end{gathered}
$$

These equations follow from direct computations. Also, the first coordinate $t^{0}$ has been privileged and considered as a time variable.

5.2. Multitime Hamilton equations. There are a lot of problems in which there is no reason to prefer one variable to the other by choosing it as time. In such cases, we use those field theories which involve many time variables (multitime or multiparameter).

In this section, we study the same functional, but instead of a single-time one, we will consider it as a multitime functional, with no privileged variable $t^{\alpha}$. We use for our computations the appropriate Hamiltonian formalism which has been given in Section 3. This formalism has been also studied and developed in [3, 4, 13, 16, 17]. The momenta associated to $L$ will be denoted by

$$
p^{\alpha \beta}=\frac{\partial L}{\partial a_{\alpha, \beta}}, \quad q_{j}^{\alpha}=\frac{\partial L}{\partial \varphi_{\alpha}^{j}}, \quad r_{j}^{\alpha}=\frac{\partial L}{\partial \eta_{\alpha}^{j}},
$$

with $\alpha, \beta=0,1,2,3$ and $j=1,2$. 
LEMMA 5.5. The momenta associated to L are

$$
p^{\alpha \beta}=\frac{1}{8}\left(a_{\alpha, \beta}-a_{\beta, \alpha}\right), \quad q_{j}^{\alpha}=2 \varphi_{\alpha}^{j}-a_{\alpha} \eta^{j}, \quad r_{j}^{\alpha}=2 \eta_{\alpha}^{j}+a_{\alpha} \varphi^{j},
$$

with $\alpha, \beta=0,1,2,3$ and $j=1,2$.

REMARK 5.6. Since the first 16 relations of Lemma 5.5 ( $p^{\alpha \beta}=\cdots$, for $\alpha, \beta=0,1,2,3$ ) are not independent, the general conditions of the Hamiltonian formalism are not fulfilled. It is yet possible to extend the Hamiltonian formalism so that such cases become workable, in various ways. One way is the following. Suppose we have a Lagrangian $L\left(t, y, y^{\prime}\right)$ such that the generalized momenta

$$
p_{i}^{\alpha}=\frac{\partial L}{\partial y_{\alpha}^{i}}
$$

are not independent. It is possible to find functions $f_{\alpha}^{i}(y, p)$ such that (5.12) be verified when we write $y_{\alpha}^{i}=f_{\alpha}^{i}(y, p)$. Suppose we made such a choice and define the formal Hamiltonian

$$
H_{f}(y, p)=f_{\alpha}^{i} p_{i}^{\alpha}-L(y, f(y, p))
$$

by treating the variables $p_{i}^{\alpha}$ as if they were functionally independent. Then we get

$$
\begin{aligned}
& \frac{\partial H_{f}}{\partial y^{i}}=\frac{\partial f_{\alpha}^{j}}{\partial y^{i}} p_{j}^{\alpha}-\frac{\partial L}{\partial y^{i}}-\frac{\partial L}{\partial y_{\beta}^{j}} \frac{\partial f_{\beta}^{j}}{\partial y^{i}}=-\frac{\partial L}{\partial y^{i}}=-\frac{\partial p_{i}^{\alpha}}{\partial t^{\alpha}} \\
& \frac{\partial H_{f}}{\partial p_{i}^{\alpha}}=f_{\alpha}^{i}+\frac{\partial f_{\beta}^{j}}{\partial p_{i}^{\alpha}} p_{j}^{\beta}-\frac{\partial L}{\partial y_{\beta}^{j}} \frac{\partial f_{\beta}^{j}}{\partial p_{i}^{\alpha}}=f_{\alpha}^{i}=\frac{\partial y^{i}}{\partial t^{\alpha}}
\end{aligned}
$$

The equations thus obtained,

$$
\frac{\partial y^{i}}{\partial t^{\alpha}}=\frac{\partial H_{f}}{\partial p_{i}^{\alpha}}, \quad \frac{\partial p_{i}^{\alpha}}{\partial t^{\alpha}}=-\frac{\partial H_{f}}{\partial y^{i}},
$$

will be named the generalized canonical equations. Note that $H_{f}$, as a function of the variables $p_{i}^{\alpha}$, depends on the choice of the functions $f_{\alpha}^{i}$, but it becomes a well-defined function on the momenta $p_{i}^{\alpha}$ by taking into consideration (5.12).

We apply this generalized Hamiltonian formalism to the functional (5.5) under the same restrictions introduced at the beginning of Section 5. The corresponding Lagrangian has been written in Lemma 5.1. The relations $p^{\alpha \beta}=(1 / 8)\left(a_{\alpha, \beta}-a_{\beta, \alpha}\right)=-p^{\beta \alpha}$ show that the momenta are not independent. In this case, we can choose the functions $f_{\alpha \beta}$ such that

$$
a_{\alpha, \beta}=f_{\alpha \beta}(u, p)=4 p^{\alpha \beta}+g_{\alpha \beta}(u, p),
$$

where $g_{\alpha \beta}=g_{\beta \alpha}$. 
LEMmA 5.7. The Hamilton density associated to $W$ and to the choice (5.16) is

$$
\begin{aligned}
H_{f}= & 2 \sum_{\alpha, \beta=0}^{3}\left(p^{\alpha \beta}\right)^{2}+\sum_{\alpha, \beta=0}^{3} p^{\alpha \beta} g_{\alpha \beta}+\frac{1}{4} \sum_{\alpha=0}^{3} \sum_{j=1}^{2}\left[\left(q_{j}^{\alpha}\right)^{2}+\left(r_{j}^{\alpha}\right)^{2}\right] \\
& +\frac{1}{2} \sum_{\alpha=0}^{3} \sum_{j=1}^{2}\left(\eta^{j} q_{j}^{\alpha}-\varphi^{j} r_{j}^{\alpha}\right) a_{\alpha}-\frac{1}{4}\left(\sum_{j=1}^{2}\left[\left(\varphi^{j}\right)^{2}+\left(\eta^{j}\right)^{2}\right]\right)^{2} .
\end{aligned}
$$

THEOREM 5.8. The Hamilton generalized equations associated to $H_{f}$ are

$$
\begin{gathered}
\frac{\partial a_{\alpha}}{\partial t^{\beta}}=4 p^{\alpha \beta}+g_{\alpha \beta}, \quad \frac{\partial \varphi^{j}}{\partial t^{\alpha}}=-\frac{1}{2}\left(q_{j}^{\alpha}+a_{\alpha} \eta^{j}\right), \\
\frac{\partial \eta^{j}}{\partial t^{\alpha}}=\frac{1}{2}\left(r_{j}^{\alpha}-a_{\alpha} \varphi^{j}\right), \quad \frac{\partial p^{\alpha \beta}}{\partial t^{\beta}}=\frac{1}{2}\left(\eta^{j} q_{j}^{\alpha}-\varphi^{j} r_{j}^{\alpha}\right), \\
\frac{\partial q_{j}^{\beta}}{\partial t^{\beta}}=\frac{1}{2} a_{\alpha} r_{j}^{\alpha}+\left(\sum_{k=1}^{2}\left[\left(\varphi^{k}\right)^{2}+\left(\eta^{k}\right)^{2}\right]\right) \varphi^{j}, \\
\frac{\partial r_{j}^{\beta}}{\partial t^{\beta}}=-a_{\alpha} q_{j}^{\alpha}+\left(\sum_{k=1}^{2}\left[\left(\varphi^{k}\right)^{2}+\left(\eta^{k}\right)^{2}\right]\right) \eta^{j}, \quad j=1,2 ; \alpha, \beta=0,1,2,3 .
\end{gathered}
$$

These equations follow from direct computations.

REMARKS 5.9. (1) In the particular case $g_{\alpha \beta}=0$, the solutions of the previous equations are subject to the conditions $\partial a_{\alpha} / \partial t^{\beta}=-\partial a_{\beta} / \partial t^{\alpha}$.

(2) From Lemma 5.5, it follows that $p^{\alpha \beta}=-p^{\beta \alpha}$. When we take into consideration these relations, any choice of the functions $f^{\alpha \beta}$ gives the same Hamilton density

$$
\begin{aligned}
H= & 2 \sum_{\alpha, \beta=0}^{3}\left(p^{\alpha \beta}\right)^{2}+\frac{1}{4} \sum_{\alpha=0}^{3} \sum_{j=1}^{2}\left[\left(q_{j}^{\alpha}\right)^{2}+\left(r_{j}^{\alpha}\right)^{2}\right] \\
& +\frac{1}{2} \sum_{\alpha=0}^{3} \sum_{j=1}^{2}\left(\eta^{j} q_{j}^{\alpha}-\varphi^{j} r_{j}^{\alpha}\right) a_{\alpha}-\frac{1}{4}\left(\sum_{j=1}^{2}\left[\left(\varphi^{j}\right)^{2}+\left(\eta^{j}\right)^{2}\right]\right)^{2} .
\end{aligned}
$$

Suitable computations give the formal Hamilton equations

$$
\begin{aligned}
& \frac{\partial a_{\alpha}}{\partial t^{\beta}}=4 p^{\alpha \beta}, \quad \frac{\partial \varphi^{j}}{\partial t^{\alpha}}=\frac{1}{2}\left(q_{j}^{\alpha}+a_{\alpha} \eta^{j}\right), \quad \frac{\partial \eta^{j}}{\partial t^{\alpha}}=\frac{1}{2}\left(r_{j}^{\alpha}-a_{\alpha} \varphi^{j}\right) \\
& \frac{\partial p^{\alpha \beta}}{\partial t^{\beta}}=-\frac{1}{2}\left(\eta^{j} q_{j}^{\alpha}-\varphi^{j} r_{j}^{\alpha}\right), \quad \frac{\partial q_{j}^{\beta}}{\partial t^{\beta}}=\frac{1}{2} a_{\alpha} r_{j}^{\alpha}+\left(\sum_{k=1}^{2}\left[\left(\varphi^{k}\right)^{2}+\left(\eta^{k}\right)^{2}\right]\right) \varphi^{j} \\
& \frac{\partial r_{j}^{\beta}}{\partial t^{\beta}}=-\frac{1}{2} a_{\alpha} q_{j}^{\alpha}+\left(\sum_{k=1}^{2}\left[\left(\varphi^{k}\right)^{2}+\left(\eta^{k}\right)^{2}\right]\right) \eta^{j}, \quad j=1,2 ; \alpha, \beta=0,1,2,3 .
\end{aligned}
$$

These are the equations from Theorem 5.8 in the particular case $g_{\alpha \beta}=0$.

(3) The first formula of Theorem 5.8 requires the integrability conditions

$$
\frac{\partial^{2} a_{\alpha}}{\partial t^{\beta} \partial t^{\gamma}}-\frac{\partial^{2} a_{\alpha}}{\partial t^{\gamma} \partial t^{\beta}}=4\left(\frac{\partial p^{\alpha \beta}}{\partial t^{\gamma}}-\frac{\partial p^{\alpha \gamma}}{\partial t^{\beta}}\right)+\frac{\partial g_{\alpha \beta}}{\partial t^{\gamma}}-\frac{\partial g_{\alpha \gamma}}{\partial t^{\beta}}=0 .
$$




\section{REFERENCES}

[1] J. P. Bourguignon, An introduction to geometric variational problems, Lectures on Geometric Variational Problems (Sendai, 1993) (S. Nishikawa and R. Schoen, eds.), Springer, Tokyo, 1996, pp. 1-41.

[2] T. de Donder, Theorie Invariantive du Calcul des Variations, Gauthier-Villars, Paris, 1935.

[3] G. Giachetta, L. Mangiarotti, and G. Sardanashvily, Covariant Hamilton equations for field theory, J. Phys. A 32 (1999), no. 38, 6629-6642.

[4] M. J. Gotay, J. Isenberg, J. E. Marsden, and R. Montgomery, Momentum maps and classical relativistic fields. Part I: covariant field theory, preprint, 1997, http://xxx.lanl.gov/ physics/9801019.

[5] J. Jost, X. Peng, and G. Wang, Variational aspects of the Seiberg-Witten functional, Calc. Var. Partial Differential Equations 4 (1996), no. 3, 205-218.

[6] J. Mawhin and M. Willem, Critical Point Theory and Hamiltonian Systems, Applied Mathematical Sciences, vol. 74, Springer, New York, 1989.

[7] Ch. Okonek and A. Teleman, Seiberg-Witten invariants and rationality of complex surfaces, Math. Z. 225 (1997), no. 1, 139-149.

[8] A. Teleman, Seiberg-Witten Theorie und komplexe Geometrie, Vorlesung, Phylosophische Fakultät II, Universität Zürich, Zurich, 1996-1997.

[9] _ Non-abelian Seiberg-Witten theory, Habilitationsschrift, Universität Zürich, Zurich, 1996.

[10] _ Non-abelian Seiberg-Witten theory and stable oriented pairs, Internat. J. Math. 8 (1997), no. 4, 507-535.

[11] A.-M. Teleman, First variation formulas for a Witten-type functional. I, An. Univ. București Mat. Inform. 50 (2001), no. 2, 247-260.

[12] _ First variation formulas for a Witten-type functional. II., An. Univ. Bucureşti Mat. Inform. 52 (2003), no. 1, 87-104.

[13] A.-M. Teleman and C. Udriște, On Hamiltonian formalisms in mathematical physics, 4th International Conference of Balkan Society of Geometers, Aristotle University of Thessaloniki, Greece, June 2002.

[14] K. Teleman, Teorie Seiberg-Witten, Curs-Anul 4, Universitatea București, Bucharest, 19951996.

[15] H. Triebel, Interpolation Theory, Function Spaces, Differential Operators, North-Holland Mathematical Library, vol. 18, North-Holland Publishing, Amsterdam, 1978.

[16] C. Udriște, Solutions of DEs and PDEs as potential maps using first order Lagrangians, Balkan J. Geom. Appl. 6 (2001), no. 1, 93-108.

[17] C. Udrişte and M. Postolache, Atlas of Magnetic Geometric Dynamics, Balkan Society of Geometers Monographs and Textbooks, vol. 3, Geometry Balkan Press, Bucharest, 2001.

[18] E. Witten, Monopoles and four-manifolds, Math. Res. Lett. 1 (1994), no. 6, 769-796.

Constantin Udrişte: Department of Mathematics I, University Politehnica of Bucharest, Splaiul Independenței 313, Bucharest 060042, Romania

E-mail address: udriste@vectron. mathem. pub.ro

Ana-Maria Teleman: Faculty of Mathematics and Informatics, University of Bucharest, Academiei 14, Bucharest 70109, Romania

E-mail address: amte1 eman@geometry.math.unibuc. ro 


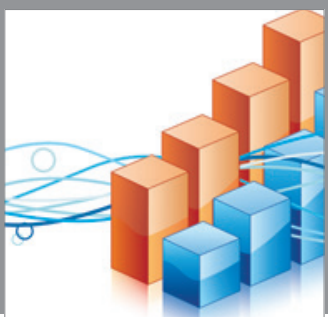

Advances in

Operations Research

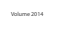

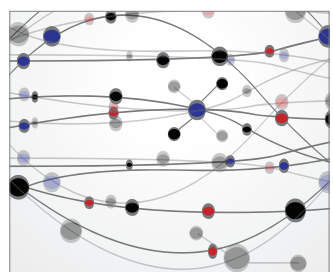

\section{The Scientific} World Journal
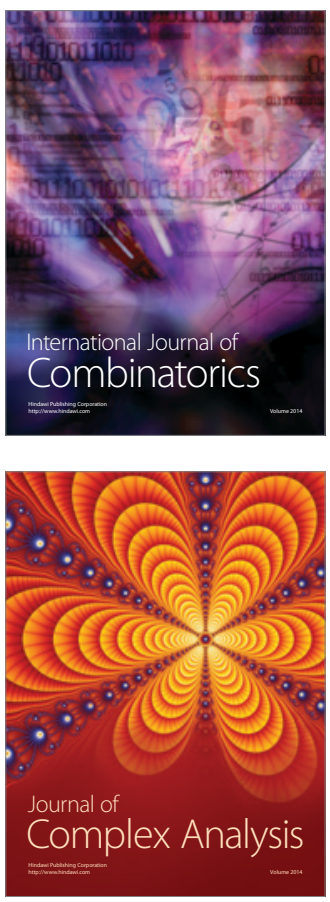

International Journal of

Mathematics and

Mathematical

Sciences
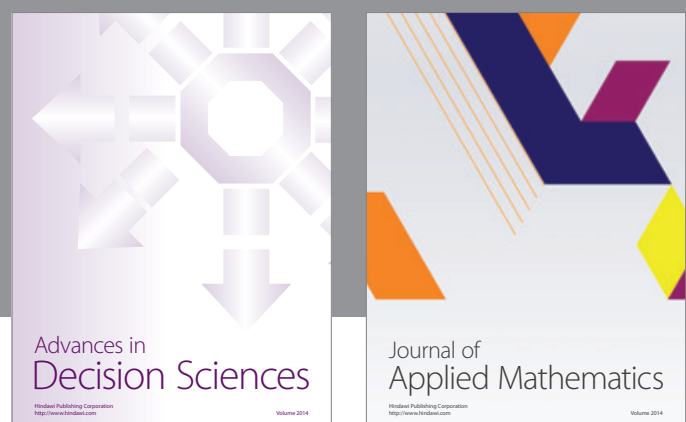

Journal of

Applied Mathematics
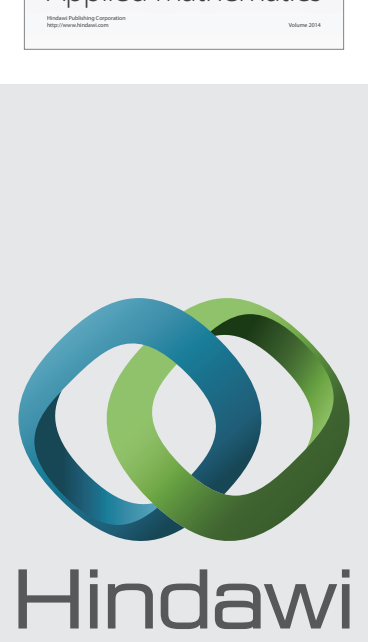

Submit your manuscripts at http://www.hindawi.com
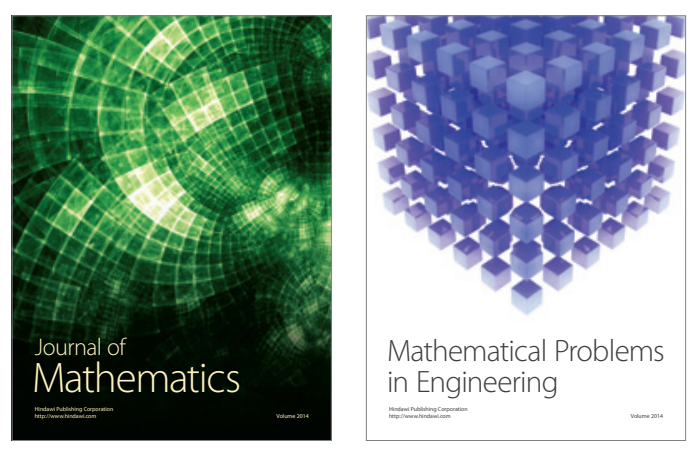

Mathematical Problems in Engineering
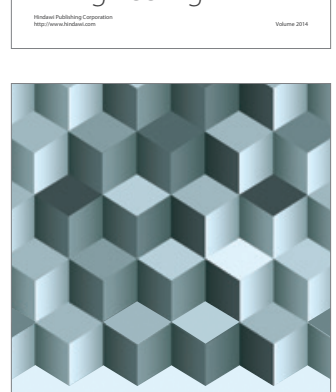

Journal of

Function Spaces
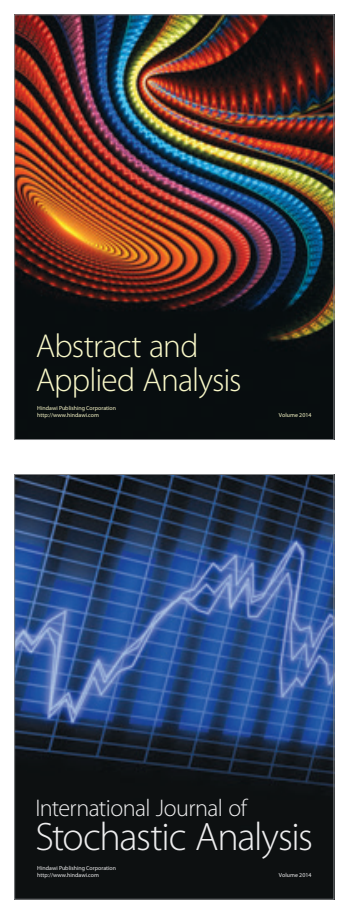

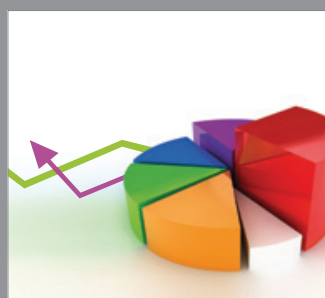

ournal of

Probability and Statistics

Promensencen
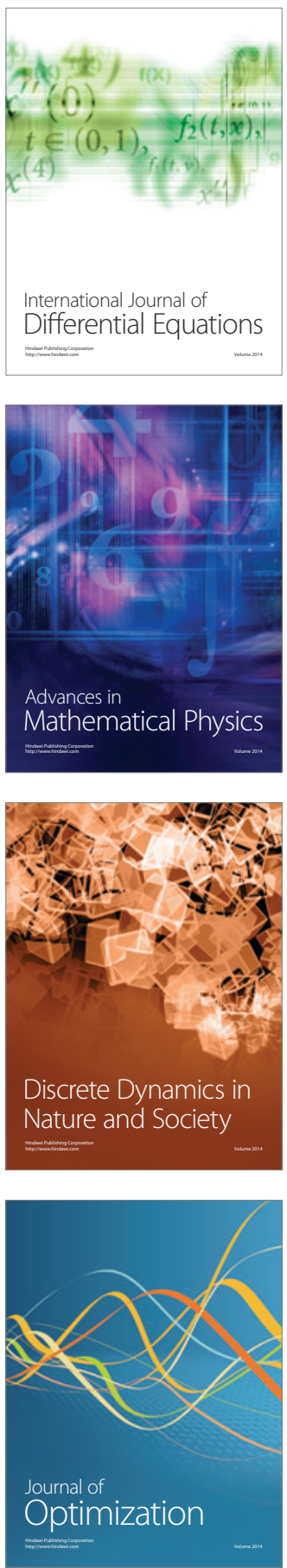